\title{
Simulation of 3D electrostatic configuration in gaseous detectors
}

\author{
N. Majumdar* and S. Mukhopadhyay \\ INO Section, Saha Institute of Nuclear Physics, \\ 1/AF Bidhannagar, Kolkata - 700064, India \\ E-mail: nayana.majumdaresaha.ac.in
}

\begin{abstract}
A Boundary Element Method (BEM) solver based on the solution of boundary integral equations of potential and electric field has been developed to simulate 3D electrostatic configuration in gaseous detectors. Use of analytical solution of the integral equations governing electric potential and field for estimating influence coefficients of the BEM solver has empowered it to provide extremely precise estimates of the potential and field for a given geometry. The nearly exact BEM (neBEM) solver has been implemented in order to simulate physical and weighting potential and field configurations in several gaseous detectors like MultiWire Proportional Counter and Time Projection Chamber. The efficacy of the solver for simulating 3D electrostatic configuration in composite systems containing both conductors and layered dielectrics has been demonstrated for some of the MicroPattern Gas Detectors and Resistive Plate Chamber. It should be noted that the method treats the dielectric interfaces to be in a steady state with polarization charges only. The reasons why the neBEM can be a preferred tool for electrostatic simulation of gaseous detectors to other 2D or 3D numerical solvers are discussed on the basis of present results.
\end{abstract}

KEYWORDS: Gaseous detectors; Detector modelling and simulations II (electric fields, charge transport, multiplication and induction, pulse formation, electron emission, etc).

${ }^{*}$ Corresponding author. 


\section{Contents}

1. Introduction 1

2. Brief descriptions of BEM and neBEM B

3. Physical potential and field distributions 7

4. Weighting potential and field distributions 7

5. Results and discussions 8

5.1 MultiWire Proportional Counter (MWPC)

5.2 MicroStrip Gas Chamber (MSGC)

5.3 MicroWire Detector (MWD) 10

5.4 microMEsh GAseous Structures (microMEGAS)

5.5 Time Projection Chamber (TPC) 13

5.6 Resistive Plate Chamber (RPC) 14

5.7 Computational resources 18

6. Conclusions 18

\section{Introduction}

A detailed study of electrostatic configuration in gaseous detectors is important to facilitate the optimization of their design as well as interpretation of their performance. Apart from the analytical approach to study the same, numerical methods like Finite Element Method (FEM) and Boundary Element Method (BEM) are widely used for this purpose. While analytical approach offers the best estimation, it can be employed only for a few simple 2D geometries. FEM is the most widely used approach because it can handle any arbitrary geometry along with dielectrics. FEM solves for the Laplace's equation at nodal points of the volume elements specified by discretization or meshing of the detector volume. However, its formulation leads to poor accuracy in estimation of potential and electric field in certain critical zones despite consuming large computational resources related to efficient meshing. The major reasons behind this loss of accuracy can be summarized in the following points.

Using a FEM solver, the solution of potential is obtained at certain predetermined nodes which is interpolated/extrapolated following a polynomial (normally of low order) to determine values at non-nodal points. Estimation of electric field is carried out by differentiating the polynomial used to represent the potential which leads to the representation of the field by an even lower order polynomial. As a result, in regions where electrostatic properties change rapidly, the electric 
field is of inadequate precision, specially at non-nodal points. Another reason for FEM not being suitable for field computation in gaseous detectors is its difficulty of treating wide variation in dimension (from microns to meters) which is common to most of these detectors. In fact, the far-field region is quite commonly artificially truncated in order to reduce computational expenses. As a result, boundary conditions at the far-field region has a possibility of being inadequately represented leading to detrimental effects on the overall solution.

BEM, on the other hand, solves boundary integral equation obtained from the solution of the Poisson's equation by numerically evaluating the potential or field as an effect of charge distribution accumulated over boundaries or material surfaces as a result of applying certain potential to the detector. The boundaries or the surfaces are discretized into a number of small elements, each carrying an unknown charge distribution. These charge distributions can be determined following Green's function technique while satisfying the given boundary condition of Dirichlet or Neumann or a mixed (Robin) type. Once the charge distributions over all the surfaces are known, the potential and electric field at any point owing to these charge distributions can be determined by using the same Green's function technique. The method is found to be more suitable for the field computation in gaseous detectors where electric field is necessary to be evaluated anywhere within the gas volume where an avalanche can take place [1]. Working on a reduced dimensional space (involving only the surfaces of the detector instead of domain volume as required by FEM), BEM can evaluate potential and electric field at any arbitrary point due to the influences of surface charge distribution on the detector boundaries with comparatively less computational expense. However, the method is less commonly used owing to the complicated mathematical exercise it necessitates in its formulation in order to handle numerical as well as physical singularities. It is also known to suffer from inaccuracies near the boundaries which is referred to as numerical boundary layer [2, 3].

In this work, we have used a new BEM solver, namely, the nearly exact BEM (neBEM) solver to estimate the electrostatic configuration of gaseous detectors. The solver is based on a novel formulation of the BEM that removes some of the major drawbacks of usual BEM. Here, the use of analytical solutions of the potential and electric field influenced by a uniform charge distribution over a rectangular 沟-6] or triangular [7, 8] boundary element allows nominally exact estimation of potential and field within the device. The analytic closed form expressions of the potential and electric field for a single boundary element have been obtained from symbolic integration of the Green's function due to uniform charge distribution over an element. As discussed in the following sections, neBEM can work on geometries with wide variation in dimensions and configurations having multiple dielectric layers without resorting to special modeling effort. Since the detailed simulation of gaseous detectors begins with the computation of electrostatic configuration within a given device and depends very critically on the accuracy of the estimated electric field at any arbitrary point, the neBEM solver is expected to become an important tool in carrying out thorough analysis of gaseous detectors. The success of the solver as illustrated below can be attributed to the advantage of the Green's function formulation for non-dissipative system coupled with the use of analytical solutions for estimating potential and electric field at any arbitrary position.

In section 2, we have briefly described the neBEM solver while in sections 3 and 4, we have discussed in brief the test cases considered in this work. In section 5, we present the results and discussions where the electrostatic configurations of six gaseous detectors have been studied. Finally in section 6, we have presented our conclusions. It may be noted here that we have compared exact 
analytic solutions against solutions obtained using the neBEM in order to illustrate the precision of the latter. Existing numerical solutions obtained using charge simulation method (2D) and using FEM (3D) have been used to illustrate the flexibility and robustness of the present solver.

\section{Brief descriptions of BEM and neBEM}

Using BEM approach, the Poisson's equation for potential

$$
\nabla^{2} \phi(\vec{r})=-\rho(\vec{r}) / \varepsilon_{0}
$$

can be solved to obtain the distribution of charges which leads to a given potential configuration. For a point charge $q$ at $\vec{r}^{\prime}$ in 3D space, the potential $\phi(\vec{r})$ at $\vec{r}$ is known to be

$$
\phi(\vec{r})=\frac{q}{4 \pi \varepsilon_{0}\left|\vec{r}-\overrightarrow{r^{\prime}}\right|}
$$

For a general charge distribution with charge density $\rho\left(\overrightarrow{r^{\prime}}\right)$, superposition holds and results in

$$
\phi(\vec{r})=\int \frac{\rho\left(\overrightarrow{r^{\prime}}\right) d v^{\prime}}{4 \pi \varepsilon_{0}\left|\vec{r}-\vec{r}^{\prime}\right|}=\int G\left(\vec{r}, \overrightarrow{r^{\prime}}\right) \rho\left(\overrightarrow{r^{\prime}}\right) d v^{\prime}
$$

where

$$
G\left(\vec{r}, \vec{r}^{\prime}\right)=\frac{1}{4 \pi \varepsilon_{0}\left|\vec{r}-\vec{r}^{\prime}\right|}
$$

is the free space Green's function for the Laplace operator in 3D with $\varepsilon_{0}$, the permittivity of free space. Similarly, the field for a general charge distribution can be written as

$$
\vec{E}(\vec{r})=-\nabla \phi
$$

leading to

$$
\vec{E}(\vec{r})=-\nabla\left(\int G\left(\vec{r}, \overrightarrow{r^{\prime}}\right) \rho\left(\vec{r}^{\prime}\right) d v^{\prime}\right)
$$

and, finally to,

$$
\vec{E}(\vec{r})=\int \frac{\rho\left(\overrightarrow{r^{\prime}}\right)\left(\vec{r}-\overrightarrow{r^{\prime}}\right) d v^{\prime}}{4 \pi \varepsilon_{0}\left|\vec{r}-\overrightarrow{r^{\prime}}\right|^{3}}
$$

The charge distribution can be obtained from equation (2.1) or (2.2) by satisfying the boundary conditions known either in the form of potential (Dirichlet) or field (Neumann) or a mixture of these two (Mixed/Robin) on material boundaries/surfaces present in the domain.

Considering the Dirichlet problem only at present (for ease of discussion), the following integral equation of the first kind can be set up.

$$
\phi(\vec{r})=\int_{\mathrm{vol}} G\left(\vec{r}, \overrightarrow{r^{\prime}}\right) \rho\left(\vec{r}^{\prime}\right) d v^{\prime}
$$

In the above equation, $\phi(\vec{r})$ is the potential at a point $\vec{r}$ in space and $\rho\left(\vec{r}^{\prime}\right)$ is the charge density at an infinitesimally small volume $d v^{\prime}$ placed at $\vec{r}^{\prime}$. The problem is, generally, to find $\rho\left(\vec{r}^{\prime}\right)$ as a function of space resulting the known distribution of $\phi(\vec{r})$. Once the charge distribution on the boundaries 
and all the surfaces are known, potential and field at any point in the computational domain can be obtained using the same equation (2.3) and its derivative.

The primary step of the BEM technique is to discretize the boundaries and surfaces of a given problem. The elements resulting out of the discretization process are normally rectangular or triangular though elements of other shapes are also used. Elements of triangular shape can be used to model geometries of any variety and, thus, is one of the most commonly used in many approaches of numerical simulation including FEM and BEM. The next step is to find out charge distribution on the elements that satisfies equation (2.3) following the given boundary conditions. The charge distribution is normally represented in terms of known basis functions with unknown coefficients. For example, in zero-th order formulations using constant basis function (collocation approach), which is also the most popular one among all the BEM formulations because of a good optimization between accuracy and computational complexity, the charge distribution on each element is assumed to be uniform and equivalent to a point charge located at the centroid of the element. This is the method that is referred to as the usual BEM in the rest of the paper. However, diverse varieties of basis function have been exercised to develop many more BEM formulations in order to represent the charge distribution on an element more efficiently so as to enhance the accuracy of the method. Since the potentials on the surface elements are known from the given potential configuration, equation (2.3) can be used to generate algebraic expressions relating unknown charge densities and potentials at the centroid of the elements. One unique equation can be obtained for each centroid considering influences of all other elements including self influence and, thus, the same number of equations can be generated as there are unknowns. In matrix form, the resulting system of simultaneous linear algebraic set of equations can be written as follows

$$
\mathbf{K} \cdot \rho=\phi
$$

where $\mathbf{K}$ is the matrix consisting of influences among the elements due to unit charge density on each of them, $\rho$ represents a column vector of unknown charge densities at centroids of the elements and $\phi$ represents known values of potentials at the centroids of these elements. Each element of this influence coefficient or capacity coefficient matrix, $\mathbf{K}$ is a direct evaluation of an equation similar to equations 2.1) or 2.2 which represents the effect of a single element on a boundary/surface (obtained through discretization) on a point where a boundary condition of the given problem is known. While, in general, this should necessitate an integration of the Green's function over the area of the element, this integration is avoided in most of the BEM solvers through the assumption of nodal concentration of singularities with known basis function. Since the right hand side of (2.4) is known, in principle, it is possible to solve the system of algebraic equations and obtain surface charge density on each of the element used to describe the conducting surfaces of the detector following

$$
\rho=\mathbf{K}^{-1} \cdot \phi
$$

Once the charge density distribution is obtained, equations (2.1) and (2.2) can be used to obtain both potential and field at any point in the computational domain.

The advantage of BEM technique in comparison to FEM may be briefly pointed out as follows. (i) It is not necessary to interpolate/extrapolate to obtain potential at non-nodal points. (ii) It is also not necessary to differentiate potential numerically to obtain field at nodal and non-nodal points. 
(iii) The boundary conditions at infinity is automatically satisfied. It is not necessary to artificially terminate the physical boundary of a problem and to devise appropriate boundary conditions at an artificial edge. In addition, since the influence coefficient matrix can be shown to depend only on the geometry of the detector, it can be inverted and stored once and for all as long as the geometry and its associated discretization does not change. Thus, for a given detector geometry, it becomes trivial to find charge distribution for different potential configurations once the matrix is obtained.

Despite the above advantages, usual BEM suffers from several drawbacks that have resulted in its relative lack of popularity. Two of the most important ones can be mentioned as follows. (i) It is assumed that a surface distribution of charge density on an element can be represented by a nodal arrangement based on a chosen basis function. (ii) It is assumed that the satisfaction of the boundary condition at a predetermined point (or, through the use of known shape functions) is equivalent to satisfying the same on the whole element in a distributed manner. The former assumption leads to infamous numerical boundary layer due to which the near-field solution in regions close to an element becomes erroneous. Thus the estimation of potential and field in nearfield region close to the boundaries and surfaces by usual BEM is found to be inaccurate. This also leads to complications in solving problems involving closely spaced surfaces such as degenerate surfaces, edges, corners and other geometrical singularities. The degenerate surface refers to a boundary, two portions of which approach each other such that the exterior region between the two portions becomes infinitely thin. It is well known that the coincidence of two boundaries gives rise to an ill-conditioned problem. A number of special formulations has been developed to cope up with these problems but, unfortunately, most of these formulations are effective in a rather small subset of problems related to potential and field that are usually faced in reality.

This problem has been resolved to a great extent through the development of the neBEM solver that uses exact integration of the Green's function and its derivative in its formulation. These integrations for rectangular and triangular elements having uniform charge density have been obtained as closed-form analytic expressions using symbolic mathematics. Thus they account for truly distributed nature of charge density on a given element. The expressions are too long and complicated to be repeated here but are easily accessible from [5-7]. Besides the fundamental change in the way the influence coefficient matrix is computed and the foundation expressions used for evaluating potential and field at any point after the charge density vector is solved for, most of the other features of neBEM are similar to any other BEM solver. The major advantage achieved through the use of the proposed closed-form expressions is that the accuracy is enhanced throughout the physical domain including near-field region without using any special formulation in any part of the domain.

In order to estimate potential and field in composite systems containing dielectric material, the above formulations have been extended following [9]. In the process, integral equations representing potential due to total charge distribution on conductor-to-dielectric $(C D)$ interfaces and polarization charge distribution (that are assumed to have attained steady state values) on dielectricto-dielectric $(D D)$ interfaces are used in conjunction with proper boundary conditions on these interfaces. It should be noted here that while the value of potential on $C D$ is usually a known quantity and constitutes the boundary condition on these interfaces, it is only the continuity of normal component of displacement vector that can be guaranteed across a $D D$ interface. It should also be noted that the presented solver is also capable of estimating effects of floating conductors as and 
when necessary. In the following, a very brief discussion on the approach which is used to solve composite systems that can be defined only by mixed boundary conditions, i.e., partly Dirichlet and partly Neumann, is presented.

Assume that there are $N_{C D}$ number of $C D$ interfaces and $N_{D D}$ number of $D D$ interfaces. The $n^{\text {th }} D D$ interface is considered to be the one where dielectric layers of permittivity $\varepsilon_{n}$ and $\varepsilon_{n-1}$ meet. It should be noted that while on each $C D$ interface, total charge $\rho_{T}$ is the sum of free charge and polarization charge, on each $D D$ interface, polarization charge constitutes the total charge. As discussed above, each interface is discretized into a set of planar rectangular and triangular elements with a constant uniform charge density distributed over each element. Superposing effect of all the elements on all the boundaries and interfaces, it can be shown that

$$
\phi(\vec{r})=\sum_{i=1}^{N_{C D}+N_{D D}} \int_{S_{i}} \frac{\rho_{T}\left(\overrightarrow{r^{\prime}}\right) d s^{\prime}}{4 \pi \varepsilon_{0}\left|\vec{r}-\overrightarrow{r^{\prime}}\right|}=\sum_{i=1}^{N_{C D}+N_{D D}} \int_{S_{i}} G\left(\vec{r}, \overrightarrow{r^{\prime}}\right) \rho_{T}\left(\overrightarrow{r^{\prime}}\right) d s^{\prime}
$$

where

$$
G\left(\vec{r}, \overrightarrow{r^{\prime}}\right)=\frac{1}{4 \pi \varepsilon_{0}\left|\vec{r}-\overrightarrow{r^{\prime}}\right|}
$$

is, once again, the free space Green's function for the Laplace operator in 3D. In the above, $S_{i}$ is the surface area of $i^{t h}$ interface and $d s^{\prime}$ is the differential element of area at $r^{\prime}$ on $S_{i}$. The electric field at any point away from an interface can be written as

$$
\vec{E}(\vec{r})=\sum_{i=1}^{N_{C D}+N_{D D}} \int_{S_{i}} \frac{\rho_{T}\left(\overrightarrow{r^{\prime}}\right)\left(\vec{r}-\overrightarrow{r^{\prime}}\right) d s^{\prime}}{4 \pi \varepsilon_{0}\left|\vec{r}-\vec{r}^{\prime}\right|^{3}}
$$

while the same for a point on an interface is

$$
\vec{E}^{ \pm}(\vec{r})=\sum_{i=1}^{N_{C D}+N_{D D}} \int_{S_{i}} \frac{\rho_{T}\left(\overrightarrow{r^{\prime}}\right)\left(\vec{r}-\vec{r}^{\prime}\right) d s^{\prime}}{4 \pi \varepsilon_{0}\left|\vec{r}-\vec{r}^{\prime}\right|^{3}} \pm \hat{n} \frac{\rho_{T}(\vec{r})}{2 \varepsilon_{0}}
$$

where $\hat{n}$ is the unit vector normal to $S_{i}$ at $\vec{r}$. The positive side of $S_{i}$ is defined as the side towards which $\hat{n}$ points to. As in single dielectric case, any given problem with multiple dielectrics can be solved by solving for $\rho_{T}$ satisfying a given set of boundary conditions.

In case of composite systems containing both conductors and dielectrics, boundary conditions are of mixed type, Dirichlet on the conductor interface $C D$ and Neumann on the dielectric interface $D D$. The potential remains a constant on each $C D$ interface and forms the Dirichlet boundary condition. Denoting the potential on $j^{\text {th }} C D$ interface by $\phi_{j}$, it can be written as follows.

$$
\phi_{j}=\sum_{i=1}^{N_{C D}+N_{D D}} \int_{S_{i}} \frac{\rho_{T}\left(\overrightarrow{r^{\prime}}\right) d s^{\prime}}{4 \pi \varepsilon_{0}\left|\vec{r}-\overrightarrow{r^{\prime}}\right|}
$$

As discussed earlier, continuity of the displacement vector across each $D D$ interface constitutes the following Neumann condition on any given $D D$ interface.

$$
\frac{\varepsilon_{j-N_{C D}}+\varepsilon_{j+1-N_{C D}}}{2 \varepsilon_{0}\left(\varepsilon_{j-N_{C D}}-\varepsilon_{j+1-N_{C D}}\right)} \rho_{T}(\vec{r})+\sum_{i=1}^{N_{C D}+N_{D D}} \int_{S_{i}} \frac{\rho_{T}\left(\overrightarrow{r^{\prime}}\right)\left(\vec{r}-\overrightarrow{r^{\prime}}\right) d s^{\prime}}{4 \pi \varepsilon_{0}\left|\vec{r}-\vec{r}^{\prime}\right|^{3}}=0
$$

where $\vec{r}$ is on $j^{t h} D D$ interface $\left(j=N_{C D}+1, N_{C D}+2, \ldots, N_{C D}+N_{D D}\right)$ under consideration and $\hat{n}$ is outward pointing unit vector. Using equations (2.8) and (2.9), it is once again possible to construct 
a system of algebraic equations as in (2.4). After obtaining total charge density $\rho_{T}$ from this set of equations, potential and field at any point for a given problem can be easily obtained by evaluating equations (2.5), (2.6) and (2.7). As in single dielectric case, the expressions in both setting up the influence coefficient matrix and subsequent evaluation of potential and field are exact solutions as discussed above.

\section{Physical potential and field distributions}

The precision, flexibility and robustness of neBEM solver have been demonstrated by simulating potential and field distributions of several gaseous detectors of diverse nature. The MultiWire Proportional Counter (MWPC) is one of the major gaseous detectors of earlier generation with many variants of it still being implemented in various experiments. The analytic solutions used for calculating electric field in a MWPC are valid for only 2D geometry [10] and thus not expected to deliver realistic estimates where the $3 \mathrm{D}$ effect is significant. The neBEM solver has been used to study in detail the potential and electric field distributions considering several geometrical variations, particularly the inclusion of side plates and change of detector length. Comparison with analytical values has been made in order to demonstrate the accuracy as well as the advantage of using a 3D solver like neBEM.

The other class of gaseous detectors studied in this work is new generation MicroPattern Gas Detectors (MPGDs) where simulation of electrostatic configuration is particularly crucial owing to their multiple dielectric configuration and presence of multiple length scales. Three popular types of MPGDs, namely MicroStrip Gas Chamber (MSGC), microMEsh GAseous Structure (microMEGAS) and MicroWire Detector (MWD) have been dealt with in order to simulate their electrostatic configurations. The accuracy of the results has been confirmed by comparing them with existing 2D BEM for MSGC [11] and with 3D FEM results for microMEGAS and MWD [12].

The presence of closely spaced surfaces in gaseous detectors with multiple dielectrics like Resistive Plate Chamber (RPC) is known to give rise to numerical complexities in conventional BEM calculation. Special formulations are devised out to handle the difficulties, whereas the neBEM does not need any such manipulation. The efficacy of the solver has been demonstrated by comparing its results to that produced by several such formulations for a multiple dielectric geometry [13]. The accuracy of the solver has been confirmed by comparing its results for weighting potential and field to the analytic solutions for configuration with one dielectric layer [14] before extending it to simulate the electrostatic configuration for a detailed realistic geometry of RPC.

\section{Weighting potential and field distributions}

The neBEM solver has as well been implemented in studying weighting potential and field in several detectors. The weighting field calculation turns out to be equally important in terms of accuracy when signal generation in a gaseous detector is studied following Shockley-Ramo theorem [15, 16]. According to the theorem, current induced on an electrode can be written as

$$
i(t)=e_{0} N(t) \overrightarrow{v_{D}}(t) \cdot \overrightarrow{E_{w}}(\vec{x}(t))
$$




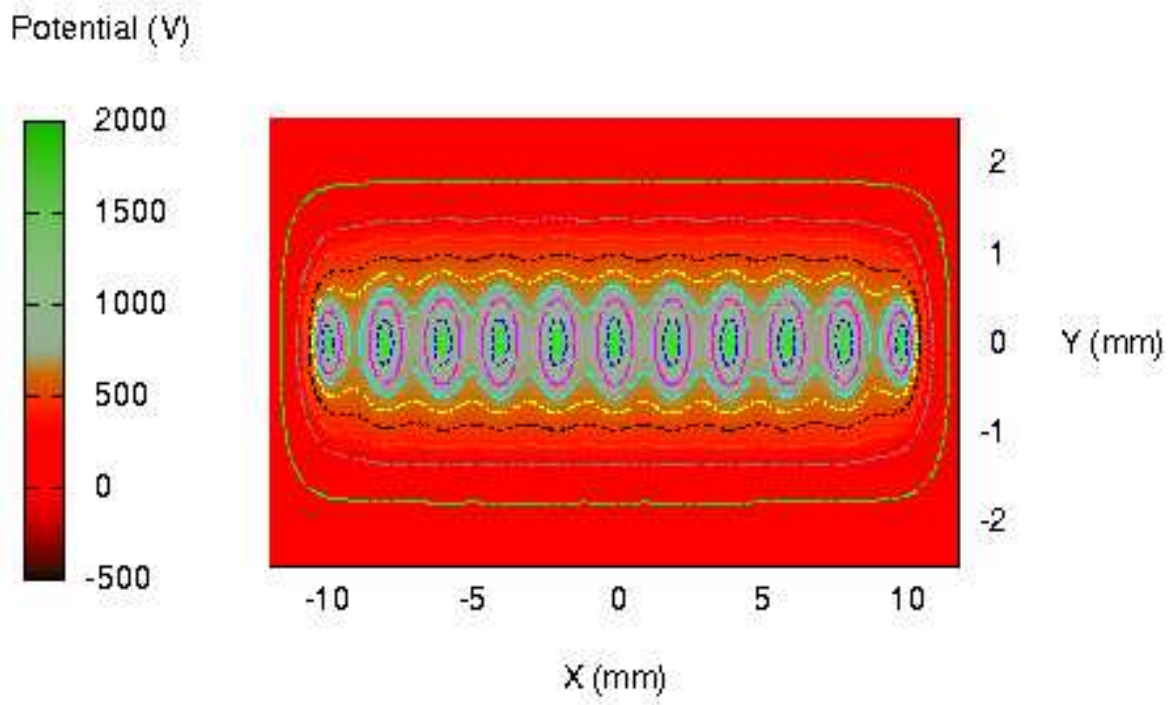

Figure 1. Potential contours on the central $X Y$-plane at $2000 \mathrm{~V}$ in MWPC

when a charge cluster $e_{0} N(t)$ moves with a drift velocity $\overrightarrow{v_{D}}(t)=\dot{\vec{x}}(t)$ where $\overrightarrow{E_{w}}(\vec{x}(t))$ is the weighting field due to specific electrode under study at a location of the cluster $\vec{x}(t)$. It is to be noted here that the weighting field can be obtained when the readout electrode is set to unit voltage keeping all other electrodes grounded.

The 3D weighting potential and field distributions have been studied in a Time Projection Chamber (TPC) for a realistic geometry. Results for a simplified 2D strip detector neglecting presence of anode wires altogether having an analytic solution are often used as an estimate for $\vec{E}_{w}$. This, however, can not be considered to be accurate for realistic TPC geometries. Another set of expressions most commonly used for design and analysis of pad chambers is obtained using conformal mapping methods and semiempirical techniques (e.g. equation (7) of [17]). While they turn out to be very useful for most of the circumstances they are limited by certain assumptions, for example, the presence of two cathode planes on either side of anode wire plane. The presence of anode wires, cathode wire plane and the effect of open or closed geometry have been studied to examine 3D effect on the weighting field configuration. A similar study has been carried out for a RPC which may be useful in simulating induced signal of the device.

\section{Results and discussions}

\subsection{MultiWire Proportional Counter (MWPC)}

A MWPC consists of an anode plane made of very thin equispaced wires. On both sides of anode, cathode planes equipped with readout pads or strips are placed. A high voltage is applied to anode plane while cathodes are maintained at ground potential. In the present calculation, geometry of the MWPC consists of 11 anode wires with diameter $20 \mu \mathrm{m}$ and pitch $2 \mathrm{~mm}$, stretched in XZ-plane. Two continuous conducting cathode plates, parallel to anode planes, have been placed at a gap of $2 \mathrm{~mm}$ with respect to anode on either sides. The dimension of the detector is $24 \mathrm{~mm} \times 4 \mathrm{~mm} \times 100 \mathrm{~cm}$, with 
length much larger with respect to other dimensions to attain 2D behavior at central $X Y$-plane. The potential contours on central $X Y$-plane across anode or cathodes are shown in figure 1 as calculated with neBEM at anode voltage $2 k V$.

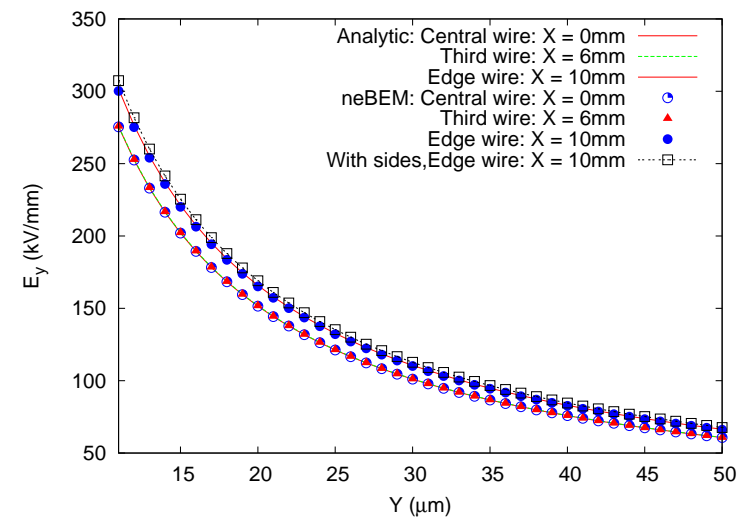

Figure 2. Normal component of electric field on the central $X Y$-plane at $2 \mathrm{kV}$ in MWPC
The variation of electric field in close proximity to anode wires has been studied with neBEM. The field values in this region are crucial since they determine avalanche size in active volume of the MWPC. The results of electric field calculated within a range of $1-40 \mu \mathrm{m}$ near an anode wire have been compared with that produced by analytic solutions using GARFIELD [10]. In figure 2, variation of normal component of electric field on central $X Y$-plane at anode voltage $2 k V$ is plotted. The values are depicted for three different wires, namely, the central, third and fifth wire (edge) on right hand side. It is evident from figure 1 that the field values are quite even (within $0.15 \%$ ) for all the wires except the edge wires where an increase of about $9 \%$ is observed with respect to the central or the third wire. The neBEM has produced an excellent agreement within $0.001 \%$ to $2 \mathrm{D}$ analytical values at centre of the geometry (for central wire) while the difference is less than $0.04 \%$ at edge.

The electrostatic field values have been studied for a wide variation of aspect ratio, $\lambda$ (length divided by breadth), ranging from 40.0 to 1.25 . However, no significant effect of aspect ratio on the field has been observed for the present geometry. To study the effect of closed geometry, grounded plates on the right, left, front and back sides of the detector geometry have been considered. The results of field calculation are same as before for all the wires except the one at edge. The values at edge for closed box geometry (illustrated by open box symbol in figure ₹) are larger by $2.4 \%$ with respect to earlier results observed in case of open geometry. The presence of side plate is presumably the reason of increase in field values observed near edge wire. The other results are not included in the figure to avoid too many data points.

\subsection{MicroStrip Gas Chamber (MSGC)}

MSGC is a detector in which wires of the standard MWPC are replaced by lithographically produced strips on solid substrates. The geometry of a typical MSGC consists of many anode and cathode strips laid alternately on a substrate. A drift volume is added before electrodes with a drift electrode placed above. High voltage is supplied to cathodes and drift electrode in order to generate a drift field. In the present calculation, 10 cathode and 9 anode strips have been considered stretched in $X Z$-plane with a pitch of $300 \mu \mathrm{m}$. The width of the strips are $100 \mu \mathrm{m}$ and $10 \mu \mathrm{m}$ respectively for the cathode and anode. Two conducting plates of dimension $2 \mathrm{~mm} \times 2 \mathrm{~mm}$, parallel to anode-cathode plane, have been included as drift plane and back plane at a distance of $3 \mathrm{~mm}$ on either sides. The cathode strips and drift plane have been provided with $-0.6 k V$ and $-1 k V$ respectively while anodes and back plane with ground potential. The potential contours on central $X Y$-plane as calculated by neBEM with a vacuum substrate (dielectric constant 1.0 ) is presented 


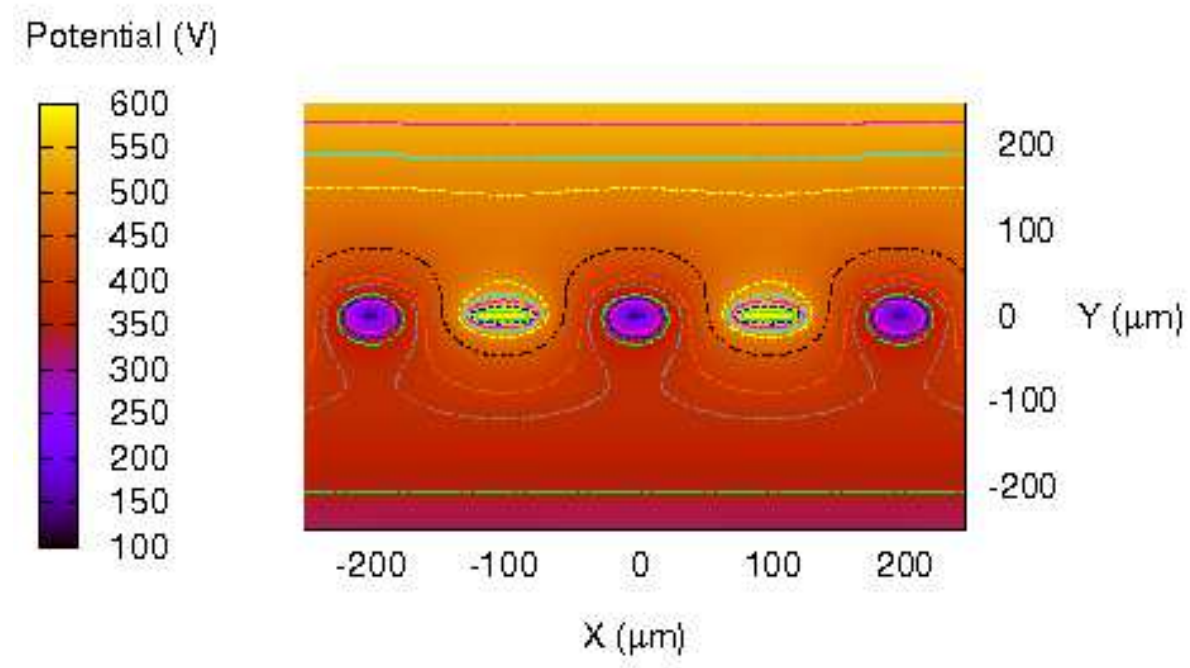

Figure 3. Potential contours on the central $X Y$-plane with a vacuum substrate in MSGC

\begin{tabular}{|c|c|c|c|}
\hline Substrate & $\begin{array}{c}\text { Thickness } \\
(\mu \mathrm{m})\end{array}$ & $\begin{array}{c}\text { neBEM } \\
(\mathrm{kV} / \mathrm{mm})\end{array}$ & $\begin{array}{c}\text { 2D [11] } \\
(\mathrm{kV} / \mathrm{mm})\end{array}$ \\
\hline Vacuum & 500 & 32.70 & 32.7 \\
& 100 & 35.47 & 35.6 \\
\hline Glass & 500 & 32.73 & 32.5 \\
& 100 & 36.91 & 36.9 \\
\hline
\end{tabular}

Table 1. Comparison of total electric field at the centre of the anode in MSGC

in figure 3. A comparative study of electric field has been made between the present results and 2D calculation following charge simulation method [11]. In the geometry, two different substrates, namely vacuum and glass (dielectric constant 5.0), have been considered each with two different thickness, $100 \mu \mathrm{m}$ and $500 \mu \mathrm{m}$. The cross-sections used in the neBEM calculation have been made identical to that used in 2D calculation in [11]. The drift plane is placed $1 \mathrm{~cm}$ away from anodecathode plane and provided with a voltage of $-3 \mathrm{kV}$. The electric field values at the centre of the anode for different geometrical conditions are tabulated in table 1. It is evident from the table that electric fields estimated by charge simulation method and by neBEM are almost identical. The maximum relative deviation is about $0.7 \%$ with respect to the earlier $2 \mathrm{D}$ calculation. This small deviation can be attributed to the facts that the present calculation is $3 \mathrm{D}$ in nature and the formulations of two methods are significantly different.

\subsection{MicroWire Detector (MWD)}

MWD is one of the various micropattern gaseous detectors that emerged with advent of printed circuit technology. It is made of a lithographically produced cathode mesh of square holes on one side of a kapton foil and anode strips on the other, running along the middle axis of cathode mesh holes. The kapton is used in a manner to provide a mechanical joint between anode strips and 


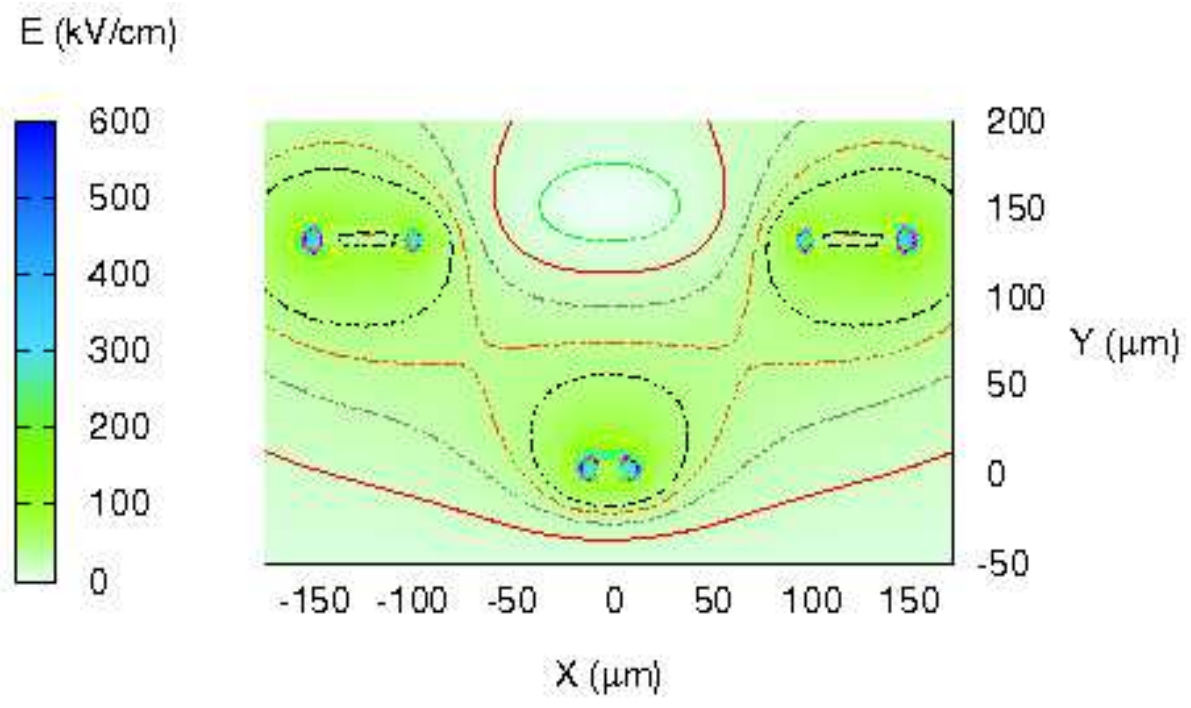

Figure 4. Total electric field contours on the central $X Y$-plane in MWD

cathode mesh of square holes. The detailed structure of this detector has been described in [12, 18]. In the present work, the same geometry as discussed in [12] has been used to pursue the calculation using neBEM, the only difference being the shape of kapton foil. It has been considered rectangular instead of trapezoidal as can be found in [12]. A drift plane has been considered $785 \mu \mathrm{m}$ away from anode strip with a voltage $-1.11 \mathrm{kV}$ following [12]. The total electric field contours in central $X Y$ plane across anode or cathode plane ( $X Y$-plane) are shown in figure 4 . The variation in total electric field along the axis passing through the mesh hole is illustrated in figure 5. The FEM calculation (using ANSYS) [12] for the same is depicted as well in the figure.

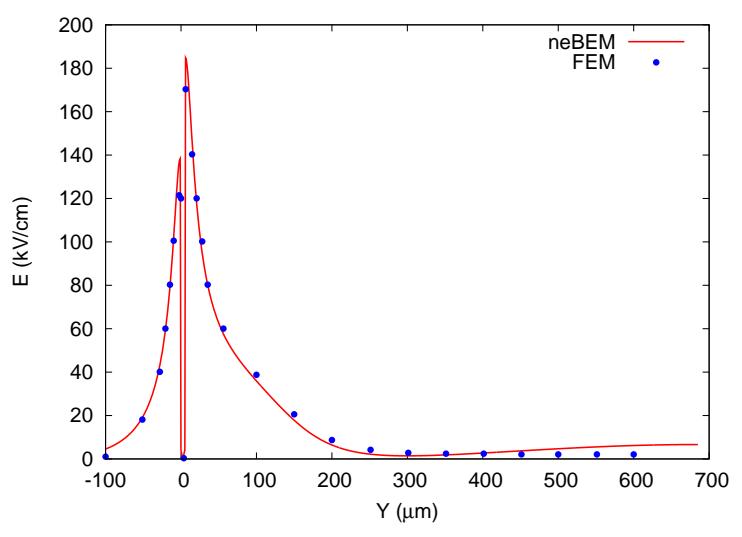

Figure 5. Total electric field along the axis of the square hole of the cathode mesh in MWD

The comparison between neBEM and FEM results yield the following. (i) Overall trend of variation is similar for both the results. (ii) In near-field region, neBEM overestimates by $8.23 \%$ (w.r.t.FEM) on positive side and by $13.22 \%$ on negative side of anode strip. (iii) In far-field region, neBEM once again overestimates electric field in comparison to FEM solution. (iv) Finally, variation in neBEM results is found to be smooth and free from the jaggedness evident in FEM solution (please refer to MWD figure centerfield.gif in [12]).

The difference between neBEM and FEM can be attributed to several factors. For example, in [12, 19] Neumann boundary condition has been used on lower boundary of FEM computational domain. Similarly, upper boundary of FEM domain has been artificially terminated by drift plane held at a constant voltage. In addition, symmetry condition on other boundaries has been assumed implying an infinite number of 


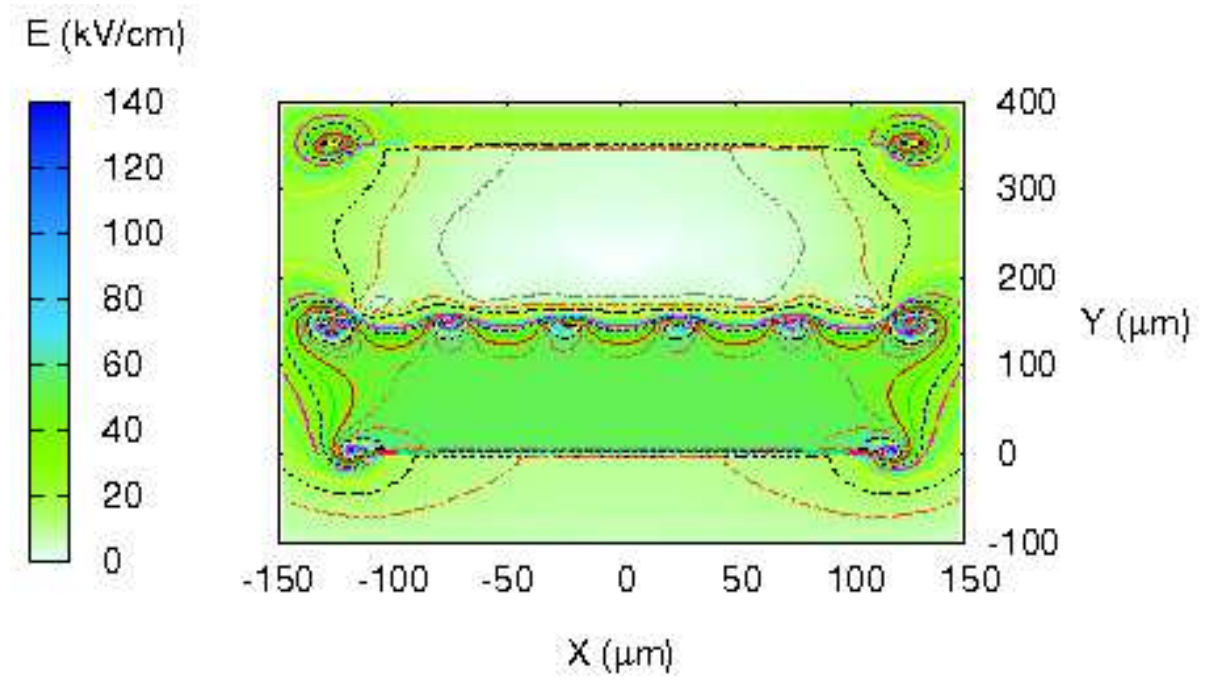

Figure 6. Total electric field contours on the central $X Y$-plane in microMEGAS

microwire units on a plane. On the other hand, in our model, we have no artificial truncation of boundary in any direction. Instead of invoking symmetry and reducing the problem to a periodic one, we have considered a $3 \times 3$ matrix of microwire units and retained 3D nature of the problem. Finally, in order to estimate potential and field at any arbitrary point, closed form analytic influence coefficients have been used without taking resort to any interpolation or extrapolation or numerical differentiation.

\section{4 microMEsh GAseous Structures (microMEGAS)}

The microMEGAS is another example of micropattern gaseous detector where a thin metal grid is used as a cathode at a very small distance above an anode readout electrode. Before the micromesh, a drift volume is added by using a drift electrode on the top. A very high electric field is generated across the gap between cathode mesh and strip. Regularly spaced supports of insulating fibers or pillars are used to guarantee uniformity of the gap. One case of microMEGAS from [12, 19] has been taken under consideration for studying its electrostatic configuration using the neBEM. The planes of anode readout and cathode mesh have been considered to lie in XZ-plane. A single anode strip of thickness $5 \mu \mathrm{m}$ placed on an epoxy substrate of dielectric constant 3.5 and cathode mesh with 7 holes across and 21 along the anode strip have been considered in neBEM calculation. A drift electrode placed $425 \mu \mathrm{m}$ away from mesh with a voltage $-0.51 \mathrm{kV}$ has been considered in order to produce a drift field $2 \mathrm{kV} / \mathrm{cm}$ approximately, following the FEM model. The contours of total electric field in central $X Y$-plane across anode or cathode plane are illustrated in figure 6 . Along axis of the mesh hole variation of electric field for several values of amplification gap is depicted in figure 7. In addition, figure 8 shows the plot of field values on anode surface as a function of amplification gap. A quantitative comparison between the two sets of results can be made from figure 8. The maximum difference between two results occurs when amplification gap is the smallest $(-5.9 \%)$ w.r.t FEM result. Two results are almost identical when the gap is in medium range while at the largest amplification gap of $85 \mu \mathrm{m}$, the difference is found to be $3.9 \%$ 


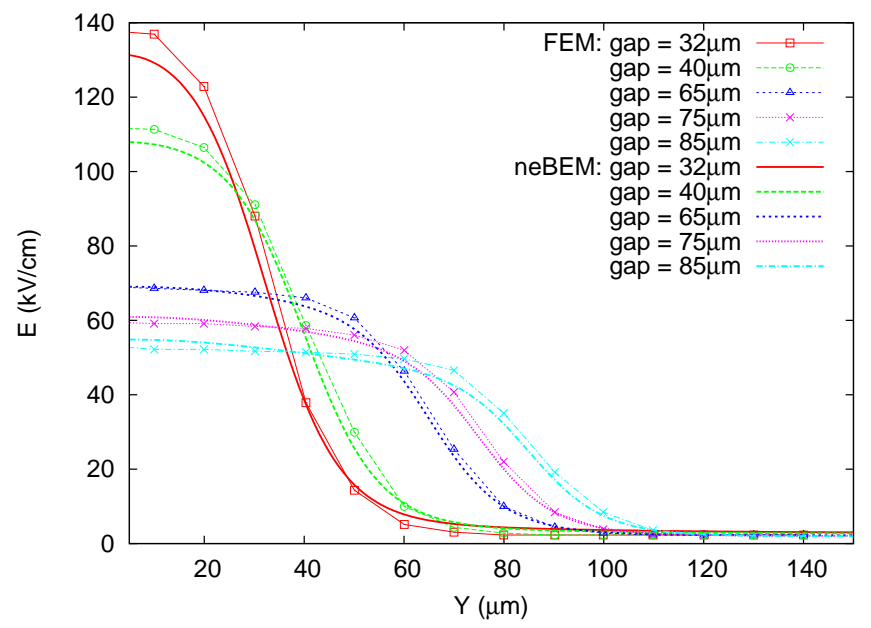

Figure 7. Normal electric field along the axis of the central mesh hole in microMEGAS

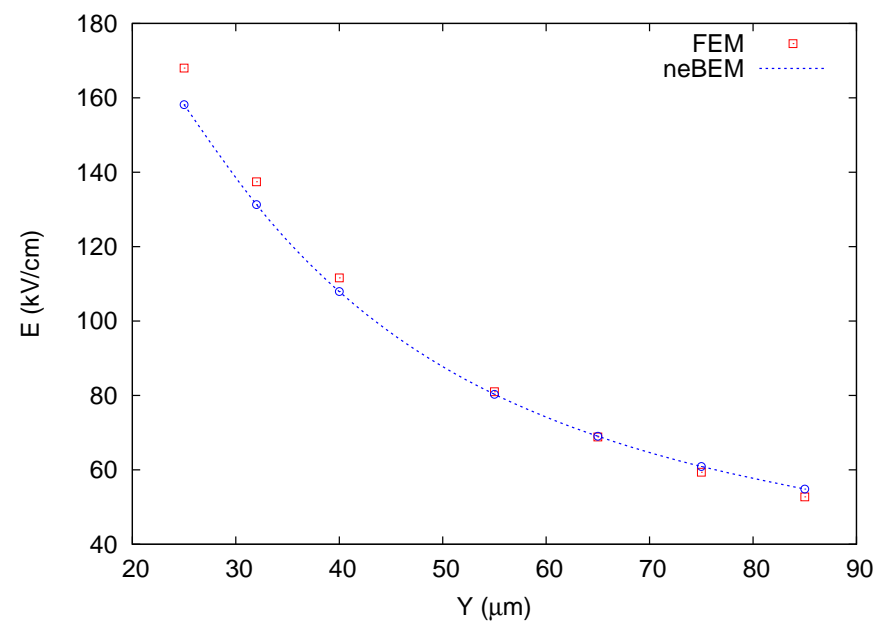

Figure 8. Normal electric field at the centre of the anode surface in microMEGAS

w.r.t FEM result. The disagreement between the values can be attributed to reasons similar to those discussed in the earlier subsection.

\subsection{Time Projection Chamber (TPC)}

The weighting potential of a TPC is normally calculated following a simple closed-form expression that exists for a 2D strip detector which consists of two conducting plates separated by a distance, $d$, and one of the plates segmented with strips of width $a$ with no gap among them [20]. It can be written as

$$
\phi(x, y)=\frac{1}{\pi}(\arctan (\tanh (\beta) \cdot \tan (\gamma))-\arctan (\tanh (\alpha) \cdot \tan (\gamma)))
$$

where

$$
\alpha=\pi \frac{x-a / 2}{2 d} ; \beta=\pi \frac{x+a / 2}{2 d} ; \gamma=\pi \frac{y}{2 d}
$$




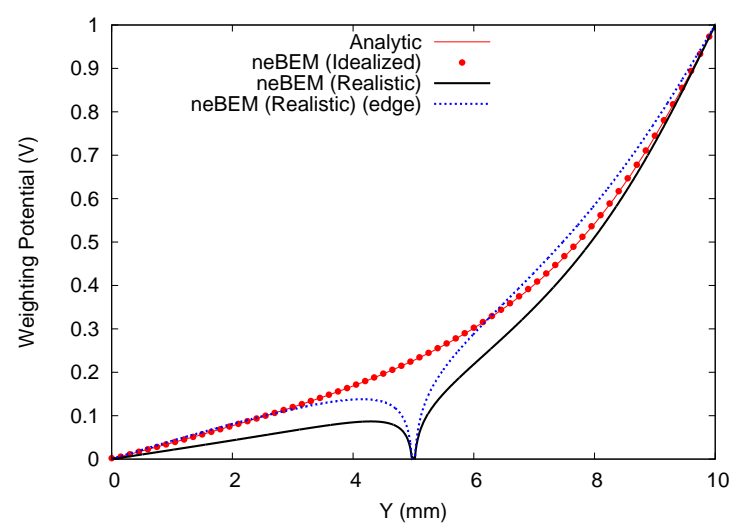

Figure 9. Weighting potential distribution on the central $X Y$-plane in TPC

The width of strip is laid along $X$-direction. This formulation neglects the presence of anode wires altogether and can not be accurate for the geometry of a realistic TPC. The weighting potential for idealized geometry of a strip detector calculated by neBEM has agreed perfectly with analytic expression. However, when a wire plane is inserted inbetween the plates, weighting potential distribution in central $X Y$-plane has been found to be significantly different throughout, the difference being very large near anode wire. It should also be noted that when upper cathode plane is replaced by a wire plane, it does not affect weighting potential distribution of figure 9 along the chosen direction passing through a grounded cathode wire. The comparison of weighting potential for idealized as well as realistic geometry with analytic solution is depicted in figure 9. In the realistic geometry, altogether 11 strips on lower cathode lying in $X Z$-plane have been considered, each having a width of $5 \mathrm{~mm}$ with no gap inbetween them. The distance between two cathode planes is $10 \mathrm{~mm}$. The dimension of each of the planes has been made $55 \mathrm{~mm} \times 15 \mathrm{~cm}$ in order to achieve 2D characteristics in the mid-section of the device. A wire plane containing 11 wires with diameter $25 \mu \mathrm{m}$ and pitch $5 \mathrm{~mm}$, each passing through middle of the strips, has been considered as an anode plane placed between the cathode planes. The upper cathode is made of similar wire plane with thick wires of diameter $100 \mu \mathrm{m}$. The 3D effect has been presented in terms of weighting potential estimates obtained at edge of the geometry $(X=2.5 \mathrm{~cm})$. The result is included in figure 9 which shows a significant edge effect.

The components of weighting electric field as per idealized and realistic geometries are illustrated in figure 10. Comparison with analytic solution demonstrates a significant departure of field values in both cases when the realistic geometry is considered.

\subsection{Resistive Plate Chamber (RPC)}

The expressions for weighting potential and electric field have been derived for an infinite plane condenser comprising of one or three homogeneous dielectric layers [14]. The analytic representation of weighting potential for a single dielectric configuration with a dielectric layer sandwiched between two conducting planes one of which is segmented into number of strips can be expressed 


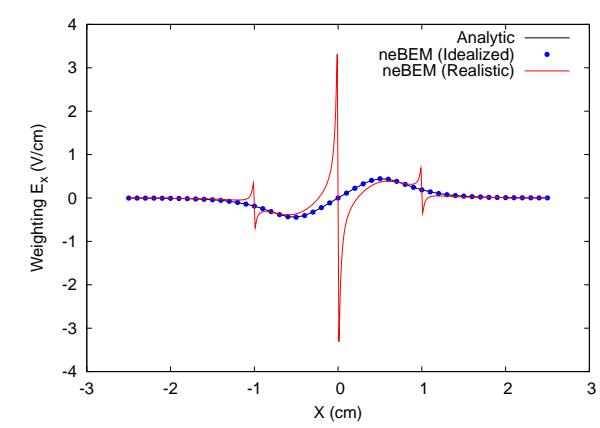

(a) Weighting field in $X$-direction

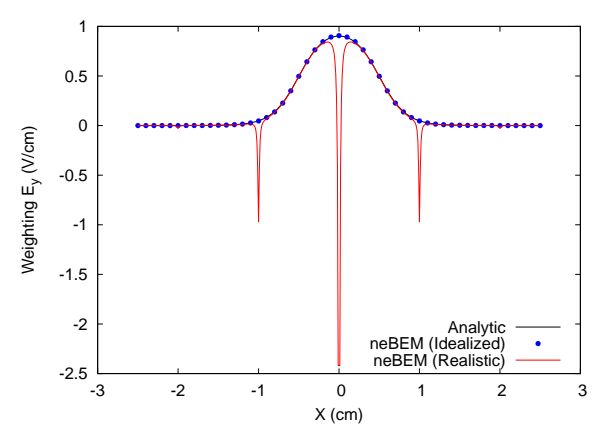

(b) Weighting field in $Y$-direction

Figure 10. Weighting electric field components on the central $X Y$-plane in TPC

as

$$
\phi(x, y)=\frac{V}{\pi}\left[\arctan \left(\cot \left(\frac{y \pi}{2 D}\right) \tanh \left(\pi \frac{x+w / 2}{2 D}\right)\right)-\arctan \left(\cot \left(\frac{y \pi}{2 D}\right) \tanh \left(\pi \frac{x-w / 2}{2 D}\right)\right)\right]
$$

where $w$ is the width of a strip along $X$-direction and $D$ the height of dielectric layer in $Y$-direction. It is assumed that strips are placed in a continuous manner with no gap inbetween them. $V$ is the potential applied to the specific strip for which weighting potential and field would be studied with all other electrodes kept at ground potential. It may be noted here that the equation (5.2) is same as equation (5.1) which is valid for a 2D strip detector. The reason of the difference between two expressions is related to geometry of the systems. In case of RPC, lower electrode is segmented whereas in TPC, it is the upper one that contains the strips. Consequently, components of the electric field can be written as

$$
\begin{aligned}
& E_{x}=\frac{V}{2 D}\left[\frac{\sin (y \pi / D)}{\cosh (\pi(x-w / 2) / D)-\cos (y \pi / D)}-\frac{\sin (y \pi / D)}{\cosh (\pi(x+w / 2) / D)-\cos (y \pi / D)}\right] \\
& E_{y}=-\frac{V}{2 D}\left[\frac{\sinh (\pi(x-w / 2) / D)}{\cosh (\pi(x-w / 2) / D)-\cos (y \pi / D)}-\frac{\sinh (\pi(x+w / 2) / D)}{\cosh (\pi(x+w / 2) / D)-\cos (y \pi / D)}\right]
\end{aligned}
$$

The solver has been validated by estimating weighting potential for a single dielectric geometry for which analytic representation of weighting potential is available from equation (5.2). The geometry has comprised of two conducting plates separated by a distance $2 \mathrm{~mm}$. Altogether 3 strips of width $3 \mathrm{~cm}$ have been considered in $X Z$-plane with length $50 \mathrm{~cm}$ in $Z$-direction. The nature of dielectric is irrelevant to mention since it has no effect on weighting potential. The comparison of weighting potential on central $X Y$-plane across dielectric layer, as calculated by neBEM, to its analytical representation following equation (5.2) is depicted in figure 11. A close agreement (within 0.0001\%) between two results has been observed.

Similarly, the variation of weighting field components along width of the strip has been compared to their analytical representations expressed in equations (5.3) and (5.4). The results are illustrated in figure 12 which demonstrates that neBEM can produce weighting field values in central plane with an accuracy about $0.0001 \%$ for a system having single dielectric layer. 


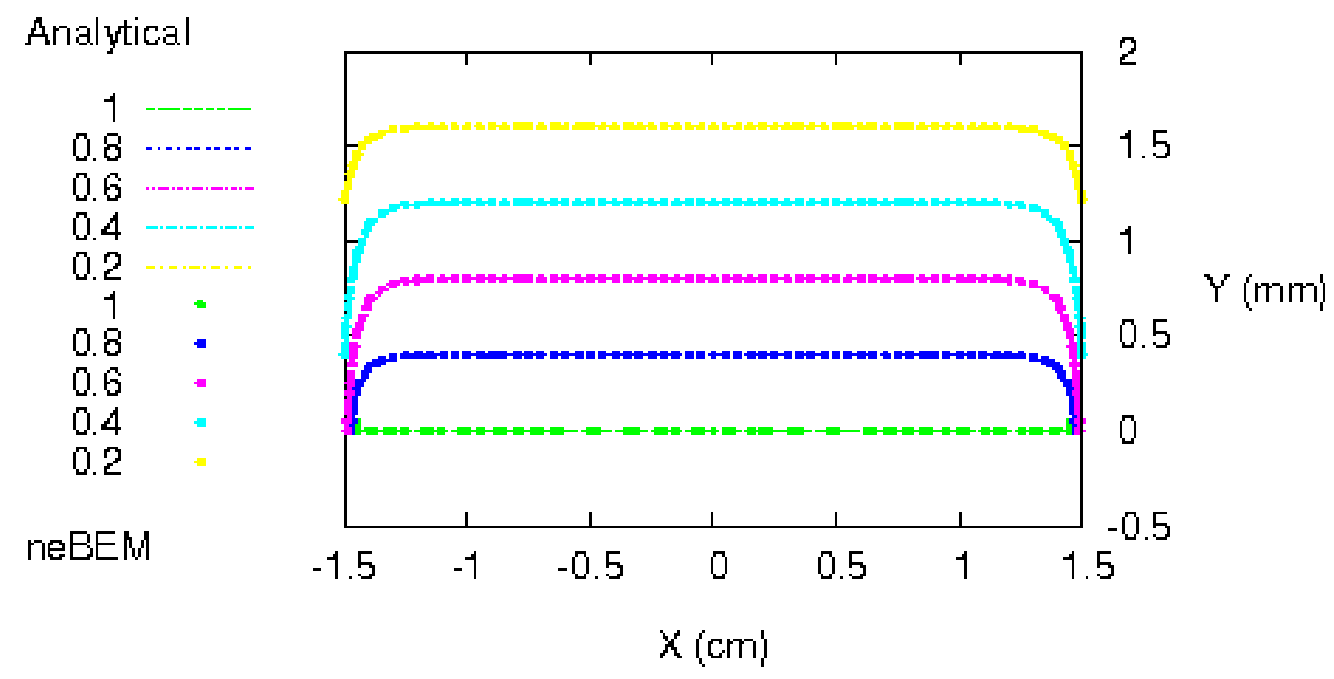

Figure 11. Weighting potential contours on the central $X Y$-plane in RPC

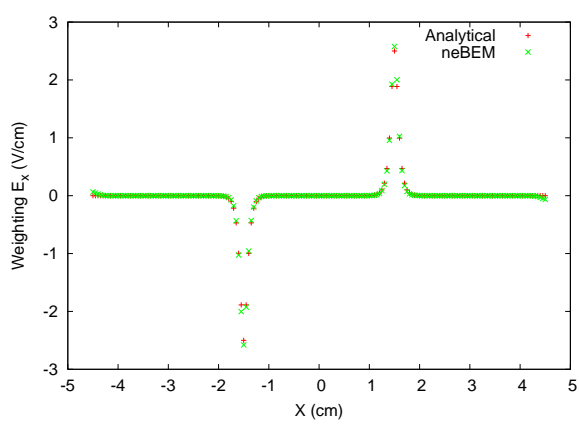

(a) Weighting field in $X$-direction

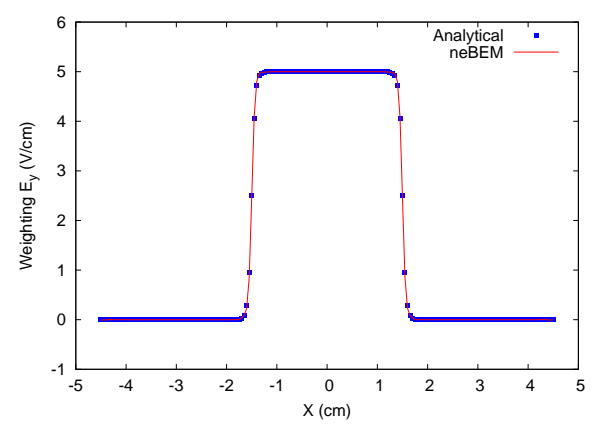

(b) Weighting field in $Y$-direction

Figure 12. Weighting electric field components on the central $X Y$-plane in RPC

A common geometry of a RPC containing more than three dielectric layers has been considered for studying its electrostatic configuration using neBEM. In the calculation, a gaseous layer (permittivity 1.000513, equivalent to Argon gas) of thickness $2 \mathrm{~mm}$ sandwiched between two glass layers (permittivity 7.75, equivalent to float glass) of same thickness has been considered. A thin coating of graphite $150 \mu \mathrm{m}$ thick has been applied on outer side of glass layers to maintain a high voltage between the layers. It should be noted here that resistivity of graphite layer is normally chosen so that this surface does not shield discharge signal from external readout strips but is small compared to the resistivity of glass to provide a uniform potential across entire surface. For calculation of weighting potential and field, graphite layer has been considered as a dielectric with permittivity 12.0 whereas it has acted as a conductor in case of physical potential and field computation. Outside graphite layers, readout strips have been considered to be lain on both sides at a distance of $100 \mu \mathrm{m}$ from graphite layer. In $X$-readout plane, total 5 strips of width $3 \mathrm{~cm}$ have been laid in $X Z$-plane with length $50 \mathrm{~cm}$ in $Z$-direction with inter-strip space $1 \mathrm{~mm}$ while the $Y$-readout 


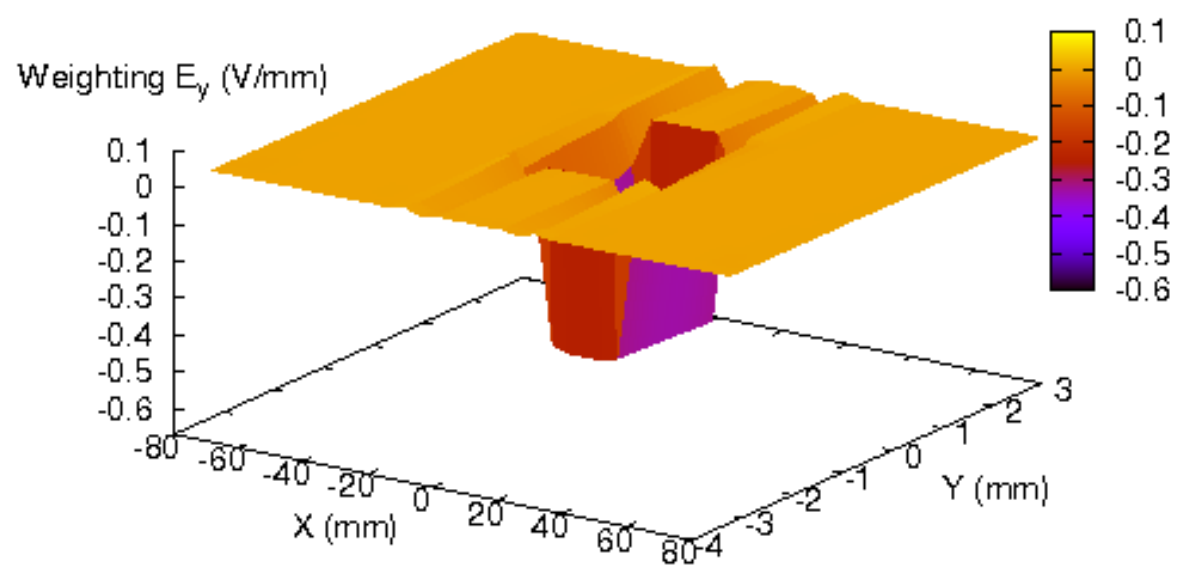

Figure 13. Normal weighting electric field on the central $X Y$-plane in RPC

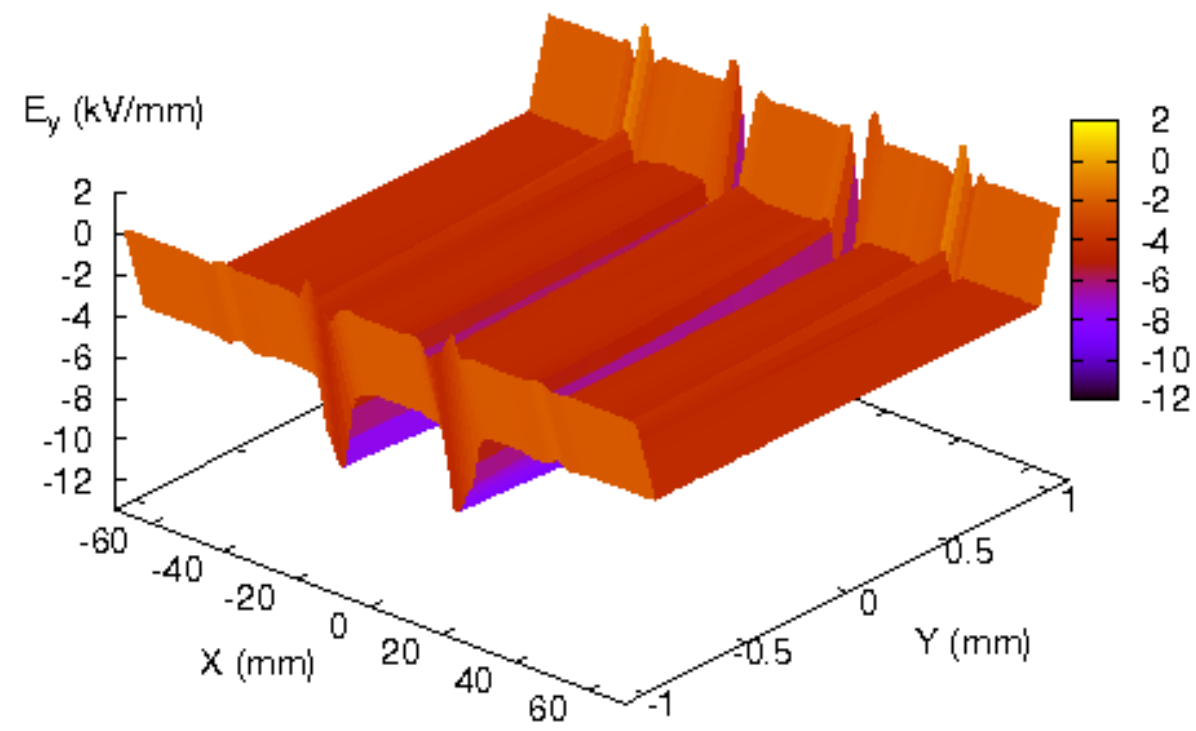

Figure 14. Physical normal electric field on the central $X Y$-plane at $8.5 \mathrm{kV}$ in RPC

plane has been considered continuous. Variation of normal component of weighting electric field on central $X Y$-plane is shown in figure 13 .

The normal component of real or physical field of realistic configuration described above has been computed with neBEM. The 3D representation of the field is illustrated in figure 14 calculated at a high voltage of $8.5 \mathrm{kV}$.

Precision of the neBEM solver has been amply demonstrated by solving the MWPC, TPC and RPC problems where we have compared the solver with exact analytic solutions. Since analytic results are derived considering $2 \mathrm{D}$ geometries, we have modeled the problems such that the two dimensionality is maintained despite the use of neBEM which is a 3D solver. Since the problems are very precisely stated while deriving analytic solutions, it has been relatively easy for us to replicate the conditions. The exact match between analytic solutions and neBEM results (dif- 
ferences $<0.01 \%$ ) with quite coarse discretization testifies to the excellent accuracy achievable using neBEM. Flexibility and robustness of the solver have been demonstrated by comparing its results with other numerical solutions, namely, charge simulation method (2D) and FEM (3D). In these cases, it is difficult to replicate computational models used in other solvers exactly because of differences in formulation and features that are characteristic of specific methods. In addition, we did not feel it necessary to make meticulous copy of the other models because the precision of the solver has already been demonstrated and it was not necessary to reproduce the exact numbers obtained using the other solvers. In these comparisons, we have tried to show that neBEM can solve complicated realistic configurations and obtain reasonable results using moderate computational resources as mentioned below.

\subsection{Computational resources}

The computation of electric potential and field of various gaseous detectors has been carried out in a 64 - bit AMD Athlon Desktop with 1.8GHz clock speed, 2.0GB RAM, running Fedora Core 5. The GNU Scientific Library has been used to carry out calculations involving complex numbers and gnuplot to generate the figures. It may be noted that we have not used any commercial packages in any part of the work.

\section{Conclusions}

Using neBEM solver, it has been possible to estimate 3D potential and electric field in several gaseous detectors. The accuracy, flexibility and robustness of the solver have been demonstrated by comparing its results with existing analytic, 2D BEM and 3D FEM results. Despite having large length scale variation $\left(1: 10^{4}\right)$ in complicated configurations having multiple dielectric layers, the solver has yielded results that are precise and reliable using commonly available computational hardware resources and free open source software. It should be mentioned here that without invoking any symmetry or using any memory or computation time saving technique in neBEM, detailed 3D results with competing accuracy could be achieved with moderately coarse discretization for all these gaseous detectors. Since detailed simulation of gaseous detector begins with computation of electrostatic configuration within the device and depends very critically on the accuracy of estimated electric field at any arbitrary point within a given device, neBEM solver is expected to contribute significantly in carrying out thorough analysis of gaseous detectors. This is even more true for new generation gaseous detectors equipped with multiscale geometry as well as various dielectrics where FEM calculation can produce erroneous results due to the use of stringent boundary conditions applied on artificially truncated boundaries that may affect near-field estimates as well. FEM estimates of potential and field for complicated geometry can also negatively be influenced by its nodal nature of solution and the necessity of polynomial interpolation and differentiation. This is even more true in the regions of interest of gaseous detector where these properties are liable to change very fast. The neBEM being a solver based on BEM can take into account the influence of all the components present in a realistic geometry and study their effect on the final electric field which leads to more reliable results. The advantage of BEM formulation coupled with the accuracy made available by analytic solutions of potential and electric field used in the neBEM has empowered this solver to excel in terms of precision and flexibility for electrostatic simulation of gaseous 
detectors compared to other existing solvers. The solver can also compute 3D weighting potential and field which facilitate simulation of charge/signal induced on any electrode of a detector due to the passage of an ionizing particle through a detector. The other relevant factors like drift velocity, avalanche multiplication etc. can be estimated using HEED, MAGBOLTZ, GARFIELD [10] which can lead to a complete simulation of the performance of any gaseous detector.

The difficulty with the present method is the solution of a fully populated matrix which can be solved by using various techniques. This aspect is under study to still reduce current computational expense.

\section{Acknowledgments}

We are grateful to Prof. B. Sinha, the Director of SINP and Prof. S. Bhattacharya, the Head of INO Section, for providing support to us to carry out this work. We thank Dr. R. Veenhof of CERN for his suggestions and help to pursue the study on TPC especially.

\section{References}

[1] S. Mukhopadhyay et al., Effect of finite dimensions of gas detectors on electric field configuration, IEEE ACM Trans. Nucl. Sc. 53 (2006) 539.

[2] V. Sladek et al., Elimination of the boundary layer effect in BEM computation of stresses, Comm. Appl. Num. Meth. 7 (1991) 539.

[3] S.-W Chyuan et al., An efficient technique for solving the arbitrarily multilayered electrostatic problems with singularity arising from a degenerate boundary, Semicon. Sci. Technol. 19 (2004) R47.

[4] S. Mukhopadhyay et al., Development of a BEM solver using exact expressions for computing the influence of singularity distributions, in proceedings of International conference on computational and experimental engineering and sciences, December, 1-6, 2005, India, TechScience Press, Forsyth, GA (ISBN 0-9717880-0-6) [physics/0604034].

[5] S. Mukhopadhyay et al., Computation of 3D MEMS electrostatics using a nearly exact BEM solver, Engg. Anal. Bound. Elem. 30 (2006) 687.

[6] N. Majumdar et al., Simulation of three-dimensional electrostatic field configuration in wire chambers: a novel approach, Nucl. Instrum. Meth. A 566 (2006) 489.

[7] S. Mukhopadhyay et al., Use of triangular elements for nearly exact BEM solutions, arXiv:0704.1563.

[8] S. Mukhopadhyay et al., Use of rectangular and triangular elements for nearly exact BEM solutions, in proceedings of International conference on emerging mechanical technology - macro to nano, 16-18 February 2007, Research Publishing, Singapore, India.

[9] M.E. Azimi and P.K. Ghosh, The numerical calculation of electrostatic environment within a dielectric composite using the boundary charge method, Modell. Simul. Mater. Sci. Eng. 2 (1994) 1093.

[10] http://garfield.web.cern.ch/garfield.

[11] S. Schmidt et al., Simulation of electrostatic properties and gas amplification in microstrip gas chambers and comparison with measurements, Nucl. Instrum. Meth. A 344 (1994) 558. 
[12] http://consult.cern.ch/writeup/garfield/examples/micropattern/,

[13] N. Majumdar et al., Computation of nearly exact 3D electrostatic field in gas ionization chambers, in proceedings of International Conference on Computing in High Energy and Nuclear Physics (CHEP06), 13-17 February 2006, India physics/0604032.

[14] T. Heubrandtner et al., Static electric fields in an infinite plane condenser with one or three homogeneous layers, Nucl. Instrum. Meth. A 489 (2002) 439.

[15] W. Shockley, Currents to conductors induced by a moving point charge, J. Appl. Phys. 9 (1938) 635.

[16] S. Ramo, Currents induced in electron motion, in proceedings of P. IRE 27 (1939) 584.

[17] E. Gatti et al., Optimum geometry for strip cathodes or grids in MWPC for avalanche localization along the anode wires, Nucl. Instrum. Meth. 163 (1979) 83.

[18] B. Adeva et al., The micro wire detector Nucl. Instrum. Meth. A 435 (1999) 402.

[19] P. Cwetanski, Studies of detector prototypes for the inner tracking system of LHCb, Diploma Thesis in Physics, CERN-THESIS-2004-007.

[20] P. Rehak et al., Detector challenges for $\mu^{+} \mu^{-}$colliders in the 10-100 TeV range, BNL (2000) 67213. 